\title{
ÉTUDE GÉNÉTIQUE DU PLUMAGE DE LA MARANS COUCOU ARGENTÉE
}

PAR

\author{
J. P. BOYER \\ Station de Recherches avicoles. \\ C. N. R. Z., Jouy-en-Josas (S.-et-O.)
}

\section{PLAN DU MEMOIRE}

Introduction

A. - Caractères liés au sexe :

I. - Analyse du coucou ;

II. - Analyse de l'argenté ;

III. - Remarque.

B. - Caractères autosomaux :

I. - Allalyse du noir ;

II. - Autres croisements ;

III. - Relations entre duvet et plumage adulte.

C. - Conclusions.

Bibliographie.

\section{INTRODUCTION}

La très grande diversité du plumage de la Marans coucou argentée nous a conduit à l'analyse des cryptomères du noir dans cette variété. D'autre part, l'inconvénient d'obtenir des animaux à souss-plumage noir nous a incité à créer, à partir de la Marans, un type d'animal herminé coucou à pattes blanches. Notre travail rentre donc dans le cadre plus général d'un programme de croisements et de sélection. Chemin faisant: nous avons cru intéressant de préciser quelques points particuliers.

Nous avons utilisé la Sussex herminée comme analyseur, de préférence à la Rhode Island rouge. Eìn effet, si cette dernière a l'avantage sur la Sussex de porter le caractère récessif " doré ", elle avait l'inconvénient d'apporter dans les croisements le gène w (patte jaune). Ceci supprimait donc la possibilité de mettre en reproduction les animaux de première génération ou reculait de quelques années l'obtention du type désiré.

L'analyse a donc dî et pu être poussée jusqu'en $\mathrm{F}_{2}$ sans inconvénient pour le programme de travail que nous nous étions fixé. 


\section{A. - CARACTERES LIES AU SEXE}

\section{I. - Analyse du coucou}

I.e standard de la Marans précise que le terme de coucou doit être compris comme un dessin caillouté ou pastillé de la plume plutôt qu'une barrure franche. Nous aurons l'occasion d'émettre quelques réserves à ce sujet.

Nous avons effectué conjointement le croisement Marans $\times$ Sussex et le croisement réciproque Sussex $\times$ Marans. Nous avons ensuite pratiqué le croisement (Rhode Island $\times$ Wyandotte) $\times$ (Marans $\times$ Sussex). Les résultats sont indiqués dans le tableau $I$.

\section{TABLEAU I}

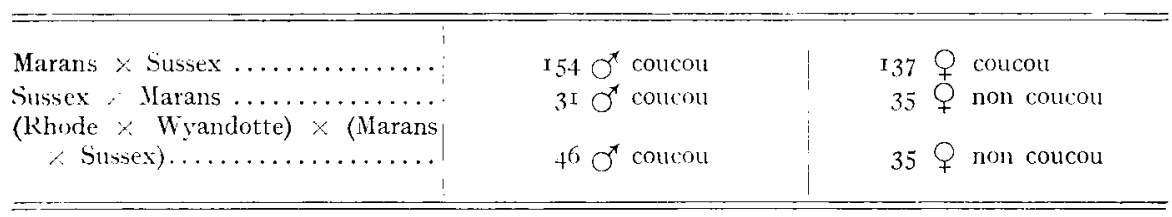

Le caractère coucou apparaît ainsi déterminé par un gène dominant et lié au sexe. Le fait qu'il s'exprime davantage chez le coq Marans que chez la poule (le mâle étant plus clair) et que les plumes du coq montrent généralement une barrure franche, nous conduit à penser que le coucou de la Marans n'est qu'une expression du gène B (Spilliman, I908). Cette hypothèse n'étant pas infirmée par les résultats observés, nous dirons que les Marans étudiés portent BB chez le coq et B- chez la poule.

\section{II. - Analyse de l'argenté}

Quatre coqs Marans ont été analysés. L'un d'eux a donné des filles dorées. Nous donnons ci-dessous les résultats (tableau II) :

TABLEAU II

\begin{tabular}{|c|c|c|c|c|}
\hline Cors Marans 9 & $9{ }_{5} 5$ souche $\mathrm{C} \ldots \ldots$ & $3^{8} \sigma^{x}$ argrentés & I5 $q$ argentées & I5 9 dorées \\
\hline & 898 & $48 \sigma^{r}$ & 409 & \\
\hline & ${ }^{9} \mathrm{I} \quad \mathrm{C} \times \mathrm{B3}$. & $37 \sigma^{x}$ & $32 ?$ & \\
\hline & HOI & $3^{\mathrm{I}} \sigma^{x} "$ & $357 "$ & \\
\hline
\end{tabular}

I ${ }^{2}$ es chiffres semblent indiquer que le coq Marans 915 n'était pas pur pour l'argenté (on notera que le standard accorde une tolérance de plumage doré) et que le doré est dû à un gène lié au sexe, récessif de l'argenté. Il est logique de penser qu'il s'agit du gène $s$ (Goodale, I9I7). 
Cette interprétation n'a pas été contredite par les résultats du croisement (Rhode Island $\times$ Wyandotte) $\times q \mathrm{~F}_{1}$ dorées. I,e coq étant Ss, si les femelles sont $s$, on doit obtenir $\mathrm{I}:$ I d'argentés et de dorés dans les 2 sexes. Or, nous avons trouvé $24 \sigma^{x}+18 \emptyset=42$ argentés et $22 \sigma^{x}+$ I7 $\uparrow=39$ dorés.

\section{III. - Remarque}

Les cartes chromosomiques donnent une distance de 63 unités entre $B$ et $s$. C'est dire que leur ségrégation est pratiquement indépendante.

Dans le cas présent, si une telle ségrégation est réalisée, l'hypothèse selon laquelle nous sommes bien en présence de $\mathrm{B}$ et s, sera éprouvée favorablement.

Nous avons noté que le croisement (Rhode $\times$ Wyandotte) $\times$ (Marans $\times$ Sussex) donnait 24 argentés +22 dorés $=46$ coqs barrés et $I 8$ argentées $+\mathrm{I} 7$ dorées $=35$ Q non barrées. Mais ceci ne préjuge en rien de la liaison Barré-doré, les poules étant hémizygotes pour ces deux gènes : $B-s$.

Nous avons donc utilisé des coqs supposés Bb, Ss, issus d'un croisement Marans $\times$ Sussex, le type parental Sussex étant bS, le type parental Marans étant supposé Bs (coqs barrés ayant quelques plumes dorées).

Le back-cross de ces mâles FI avec des poules Sussex a donné les résultats suivants chez les femelles (tableau III), le cas des coqs étant ici sans intérêt, puisqu'ils expriment tous 1'argenté. Soit $\%^{2}=4,37$ d'où $\mathrm{P}=0,20-0,25$ dans l'hypothèse d'une ségrégation indépendante. On notera que le type parental Bs est précisément le moins représenté.

\section{TABLEAU III}

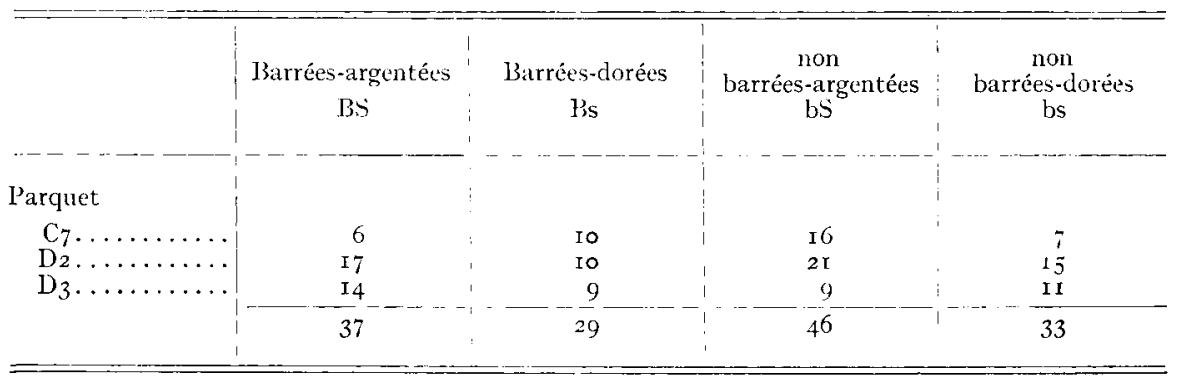

B. - CARACTERES AUTOSOMAUX

\section{I. - Analyse du noir}

Quatre coqs Marans ont été analysés en utilisant le procédé du "double-schift ": deux coqs sont croisés successivement avec les mêmes poules Sussex, un intervalle de trois semaines étant respecté entre le 
retrait du premier coq et le ramassage des œufs à couver du deuxième. Les résultats sont portés dans le tableau IV.

TABLEAU IV

\begin{tabular}{|c|c|c|c|c|}
\hline & $\sigma^{x}$ noirs & $\sigma^{x}$ herminés & q noires & $q$ herminées \\
\hline \multicolumn{5}{|l|}{ Parquet $\mathrm{B}_{9}$} \\
\hline $\begin{array}{l}\mathbf{I}^{\mathbf{0}} \operatorname{coq} \ldots \ldots \ldots \\
2^{\mathbf{0}} \operatorname{coq} \ldots \ldots \ldots\end{array}$ & $\begin{array}{l}18 \\
37\end{array}$ & $\begin{array}{c}20 \\
0\end{array}$ & $\begin{array}{l}\mathrm{I}_{4} \\
3^{2}\end{array}$ & $\begin{array}{l}16 \\
0\end{array}$ \\
\hline \multicolumn{5}{|l|}{ Parquet Bio } \\
\hline $\begin{array}{l}\mathrm{I}^{\mathrm{O}} \mathrm{coq} \ldots \ldots \ldots \ldots \\
2^{0} \operatorname{coq} \ldots \ldots \ldots \ldots\end{array}$ & $\begin{array}{l}48 \\
\text { I } 2\end{array}$ & $\begin{array}{l}\circ \\
\text { I0 }\end{array}$ & $\begin{array}{l}40 \\
20\end{array}$ & $\begin{array}{l}\circ \\
15\end{array}$ \\
\hline
\end{tabular}

Les résultats du $2^{\mathrm{e}} \operatorname{coq} \mathrm{d} u$ Bg et du I ${ }^{\text {er }} \operatorname{coq}$ du BIo indiquent que le noir de la Marans est dominant sur l'herminé de la sussex. Il s'ensuit vraisemblablement que le $\mathrm{I}^{\mathrm{er}} \operatorname{coq} \mathrm{du} \mathrm{B} 9$ et le $2^{\mathrm{e}}$ du BIo étaient hétérozygotes. L'herminé étant dî au gène $e$ (Lippinco'Tr', I9I8), les Marans utilisés portaient soit IiL; soit Eie.

Les animaux herminés Ii s semblaient donc être ee. Nous avons effectué pour vérification la Frz issue du croisement Fi herminé $\times I_{1}$ herminé. Or,

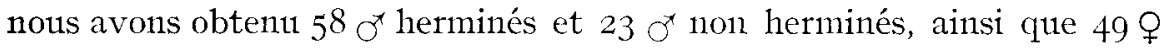
herminées et $2 \mathrm{I}$ \& non herminées. I e phénotype "non herminé 》 correspond à un plumage gris plus ou moins foncé, assez voisin du type Marans chez les cocs. Les poules étaient en outre fortement saumonées.

On peut interpréter cette F'z dans l'hypothèse d'un seul gène, récessif de $e$. La ségrégation $3 / \mathrm{I}$ donne en effet un $\%^{2}=\mathrm{I}, 37$ d'où $\mathrm{P}=0,2-0,3$. Il s'agirait par conséquent d'un allèle de $\mathrm{E}$ et de $e$, récessif de ces deux gènes. On peut penser qu'il s'agit du gène $e^{+}$(KImbalL, I952). Deux des coqs Marans analysés étaient par suite EE et deux autres $\mathrm{E} e$.

Sans nous arrêter sur des chiffres, nous indiquerons quelques faits à l'appui de cette interprétation.

Chez divers individus issus des différents croisements - et plus précisément chez les poules - nous avons noté les dessins de plume suivants (tableau $\mathrm{V}$ ).

\begin{tabular}{|c|c|c|}
\hline Dessin & Région & Attribuable à \\
\hline$--\cdots---$ & $\ldots \ldots$ & $-\cdots-------\cdots$ \\
\hline $\begin{array}{l}\text { Pointille } \ldots \ldots \ldots \ldots \ldots \ldots \ldots \ldots \ldots \\
\text { Crayonné } \ldots \ldots \ldots \ldots \ldots \ldots \ldots \ldots \ldots \ldots \\
\text { Tache apicale } \ldots \ldots \ldots \ldots \ldots \ldots \ldots \ldots\end{array}$ & $\begin{array}{l}\text { Toos } \\
\text { Poitrine } \\
\text { Camail-dos }\end{array}$ & $\begin{array}{l}\text { Sg (Stipplingr) } \\
\text { Pg (l'encilling) } \\
\text { As (Apical Spangling) }\end{array}$ \\
\hline
\end{tabular}

A l'exception de la tache apicale As, qui - à l'état homozygote est épistatique au noir $\mathrm{E}$, le pointillé et le crayonné se manifestent essentiellement en présence de $e^{+}$(KImbal,L, I953). 
Néanmoins, il demeure possible que le gène incriminé ne soit pas $e^{+}$, mais un quatrième ou cinquième allèle de E. Les recherches dans ce sens sont assez avancées, mais les neuf allèles de la série $\mathrm{E}$ diagnostiqués par Krmbali (1954) ne sont pas tous encore mis en évidence.

Jusqu'à plus ample information, nous garderons donc 1'hypothèse de la présence du gène $e^{+}$chez les Marans (en même temps que celle de $\mathrm{E}$ ), ou du moins, d'un gène allèle de $\mathrm{E}$ et de $e$, récessif de tous deux.

\section{II. - Autres croisements}

I. - Afin d'éprouver ces conclusions, nous avons effectué le croisement en retour $\sigma^{*} F_{I}$ type Marans $\times \propto$ sussex, les $\sigma^{r}$ Fi étant issus du croisement Marans $x$ sussex, les résultats sont indiqués dans le tableau VI.

\section{TABLEAU VI}

\begin{tabular}{|c|c|c|c|c|}
\hline $\begin{array}{l}\text { Fi Type } \\
\text { Marans } \times \text { Sussex }\end{array}$ & $\begin{array}{c}\text { Noir barré } \\
\text { liB }\end{array}$ & $\begin{array}{l}\text { Noir non barrí } \\
\text { lib }\end{array}$ & $\begin{array}{c}\text { Herminé barré } \\
\mathrm{eI}\end{array}$ & $\mid \begin{array}{c}\text { IIerminé non barrê } \\
\text { eb }\end{array}$ \\
\hline \multicolumn{5}{|l|}{ Parquet } \\
\hline 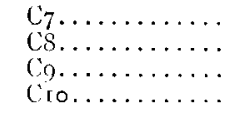 & $\begin{array}{l}\text { I } 2 \\
\text { I } 7 \\
16 \\
20\end{array}$ & $\begin{array}{l}16 \\
17 \\
1.3 \\
18\end{array}$ & $\begin{array}{l}18 \\
20 \\
19 \\
19\end{array}$ & $\begin{array}{l}29 \\
23 \\
22 \\
26\end{array}$ \\
\hline Total ..... & 65 & 64 & 76 & 100 \\
\hline
\end{tabular}

Il semble bien, d'après ces résultats, que les coqs Fi I utilisés étaient $\mathrm{E} e$, mais le fait d'une ségrégation indépendante conduit à $\mathfrak{u n}^{2} \%=\mathrm{II}, \mathrm{O} 3$ d'oì $\mathrm{P}=0, \mathrm{OI}-\mathrm{O}, 02$. Nous émettrons l'hypothèse de l'action sublétale de $\mathrm{E}$, en accord avec les travaux de HUTT ( $195 \mathrm{I}$ ). Le tableau VII indique les $\%$ et les probabilités correspondantes suivant les deux interprétations.

TABLEAU VII

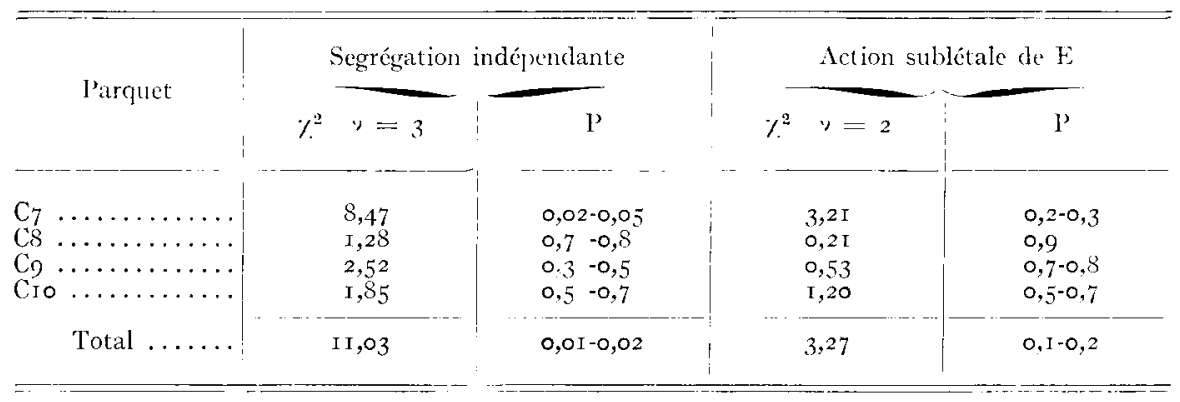

Bien qu'à part le parquet $C_{7}$, l'hypothèse de la ségrégation $x: x$ :I :I soit acceptable, les animaux de phénotype $\mathrm{E}$, sont systématiquement les 
moins nombreux. I'ajustement apparaît bien meilleur suivant la seconde hypothèse.

Notons qu'à l'inverse de HuTr', nous n'avons pas trouvé que la barrure liée au sexe empêchait l'action létale de $\mathrm{E}$; mais nous n'avons pas non plus noté la présence d'une zone dénudée (bare-back) chez les poussins, bien que le duvet soit plus court chez l'animal portant $\mathrm{E}$.

I es parquets $\mathrm{C} 8$ et Cro donnent un ajustement tout à fait satisfaisant dans 1'hypothèse de la ségrégation indépendante. Ceci nous conduit à penser qu'il est possible de disjoindre l'action létale de E de son action mélanisante. Autrement dit, le gène E n'aurait pas une action pléiotropique, mais serait lié fortement à un gène sublétal.

2. - Nous avons d'autre part obtenu une $F_{2} 2$ à partir du croisement $\sigma^{\top}$ FI herminé $\times \subsetneq$ FI type Marans. Ces animaux étaient issus des croisements Marans $\times$ Sussex indiqués précédemment, les coqs étant par suite $e e^{+}$et les poules Ee. I es résultats sont figurés dans le tableau VIII.

\section{TABLEAU VIII}

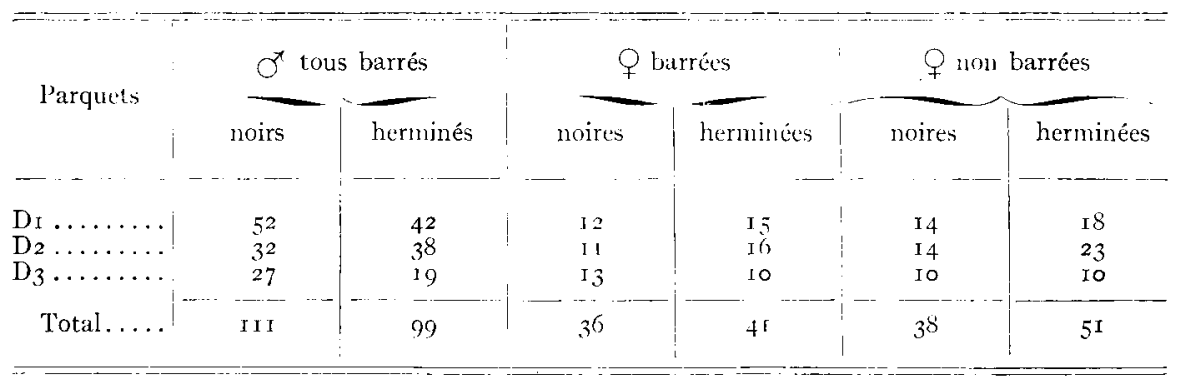

L'hypothèse de la ségrégation I : I est acceptable en ce qui concerne le noir et 1 'herminé $\left(\psi^{2}=0,094-\mathrm{P}=0,7-0,8\right)$. En appliquant cette même hypothèse aux poules seulement, en ce qui concerne les quatre catégories de phénotypes (1e cas des coqs, exprimant tous $B$, est ici sans intérêt), on trouve un $\%^{2}=3,2$ I soit $\mathrm{P}=0,3-0,5$ avec $\%=3$.

Selon l'hypothèse de l'action sublétale de $\mathrm{E}$, on trouve $\%^{2}=2,22$ soit $\mathrm{P}=0,3-0,5$ également (avec $\%=2$ ). L,es deux interprétations sont donc plausibles.

\section{III. - Relation entre duvet et plumage adulte}

Les relations entre duvet et plumage adulte semblent complexes. Des poussins à duvet clair ont donné des adultes type Marans. Inversement, des poussins à duvet foncé, ont donné des adultes pratiquement herminés, la région du dos étant souvent garnie de plumes pointillées, 
ou tachées et même chez les poules, saumonées. Le croisement $\sigma^{*}$ herminé $\times$ OFI type Marans - coqs et poules étant issus des croisements Marans $\times$ Sussex - a donné les résultats suivants (tableau IX).

TABLEAU IX

\begin{tabular}{|c|c|c|}
\hline Duvet & Plumage noir & l'lumage herminé \\
\hline $\begin{array}{l}\text { Noir ou fortement rayé } \ldots \ldots \ldots \\
\text { Blanc uni ou gris fumé } \ldots \ldots \ldots\end{array}$ & $\begin{array}{r}\mathrm{I} 80 \\
5\end{array}$ & $18 \frac{4}{5}$ \\
\hline
\end{tabular}

N. B. - I,es adultes aberrants provenaient de poussins noirs et blanc uni.

Le recroisement $\sigma^{x}$ FI type Marans $x$. Sussex, les coqs étant issus des croisements Marans $\times$ Sussex, a donné des résultats un peu différents : aucun poussin blanc uni ou gris fumé n'a donné d'adulte noir (tableau X).

\section{TABLEAU $\mathrm{X}$}

\begin{tabular}{|c|c|c|}
\hline Duvet & Plunage noir & Plumage herminé \\
\hline $\begin{array}{l}\text { Joir ou fortement rayé ....... } \\
\text { Blanc ou gris .............. }\end{array}$ & I 29 & $\begin{array}{r}\text { I } 2 \\
\mathbf{1 6 4}\end{array}$ \\
\hline
\end{tabular}

N. B. - I.es adultes aberrants provenaient de poussins noirs.

Ennfin, le croisement $\sigma^{r}$ Fi herminé $\times q$ FI herminée - coqs et poules étant issus des croisements Marans $\times$ Sussex — a donné également des résultats différents, les poussins gris donnant tantôt des adultes noirs, tantôt des adultes herminés (tableau XI).

\section{TABLEAU XI}

\begin{tabular}{|c|c|c|}
\hline Duvet & Plumage noir & Plumage herminé \\
\hline 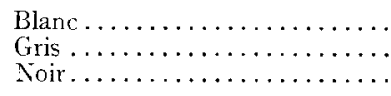 & $\begin{array}{r}5 \\
19 \\
24\end{array}$ & $\begin{array}{r}51 \\
44 \\
.\end{array}$ \\
\hline
\end{tabular}

Si l'on attribue à chaque phénotype une certaine valeur - par exemple $-I$, zéro et $+\mathrm{I}$ aux duvets noirs, gris et blancs; o et $+\mathrm{I}$ aux plumages noirs et herminés, on trouve une corrélation $r=+0,5 \mathrm{I}$. Ia liaison semble donc loin de la perfection. Bien que l'on puisse 
incriminer les erreurs d'observation, celles-ci seraient grossières. Notons plutôt que le plumage peut n'avoir aucun rapport avec le duvet, alors que le sous-plumage est en général de la couleur du duvet, avec néanmoins des exceptions (Cochez, I954). Nous avons effectivement observé des animaux herminés à sous-plumage coloré, gris ardoise, et des animaux type coucou, assez clairs d'ailleurs, à sous-plumage carrément blanc. Mais les chiffres nous manquent, divers documents ayant été détruits accidentellement.

En ce qui concerne les poussins blanc uni donnant des adultes colorés, nous pensons plutôt à la présence dans les croisements d'un facteur de coloration retardée. Celui-ci semble présent chez la sussex (PEAsr et Cock, I95I). Nous avons d'autre part constaté quelques cas de poussins Sussex purs dont le duvet n'était pas entièrement blanc, mais très légèrement taché de gris sur la tête et le dos. Le gène Wh (Wheaten) apporté par la Sussex expliquerait ainsi l'existence de poussins blancs donnant des adultes colorés. Rappelons en passant que tel est le cas normal chez la Fiaverolles saumonée, signalé par l'ÉASE et KIMBarl et observé dans nos souches.

Mais inversement, plusieurs poussins noirs ou gris ont donné des adultes herminés. Un fait analogue fut noté par LiIPINcotT (I92I) chez la Wyandotte et la Plymouth Rock, fait confirmé par Sinru. Cochrz a retrouvé un cas semblable dans u11 croisement Marans noir cuivré $\times$ Wyandotte blanche. I,es résultats n'ont pas encore été publiés.

Nous serions ainsi en présence de deux facteurs différents, l'un de coloration retardée, probablement Wh, apporté par la sussex, 1'autre de décoloration retardée, apporté par la Marans.

\section{C. - CONCLUSIONS}

I. - Le standard de la Marans coucou argentée ne nous parât pas satisfaisant à divers points de vue. Tout d'abord, la tolérance de plumage doré revient sans doute à mélanger cette variété avec la variété dorée. Fn général, set1l le camail des Marans coucou dorées est effectivement doré, les barrures des autres plumes du corps étant blanches (génotype $\mathrm{EE} \mathrm{B} \mathrm{-} \mathrm{s}-$-). Il convient toutefois de ne pas confondre ce doré avec la couleur paille que l'on peut rencontrer dans les lancettes et sur le dos des coqs argentés et qui seule doit être tolérée. D'autre part, le terme de coucou devrait être entendu comme une barrure franche. Il est en effet vraisemblable de penser que la présence du noir $e^{+}$est assez fréquente chez la Marans et que le caillouté et le pastillé sont des manifestations de génotypes variés contenant $e^{+}, \mathrm{Sg}, \mathrm{Pg}$ et $\mathrm{B}$ à diverses doses. On notera également qu'il existe deux variétés de Marans - l'une dite saumonée perdrix, 
à peu près disparue, l'autre noire cuivrée, dont la description correspond nettement à un génotype $e^{+} e^{+}$. Enfin, rappelons que la Marans compte parmi ses ancêtres divers combattants anglais, ce qui explique la très grande variabilité de son plumage, et qu'à l'exception du gène $B$, épistatique à $\mathrm{E}$, de nombreux gènes secondaires du plumage, tels que $\mathrm{Sg}, \mathrm{Pg}$ et As, ont pu être "noyés " sous l'épistasie de F apporté par la Langshan. Dans ces conditions, la Marans coucou argentée apparait loin d'être fixée et l'analyse des cryptomères du noir E est nécessaire.

2. - Des quelques analyses que nous avons réalisées, nous pouvons tirer les conclusions suivantes :

- I a Marans coucou argentée porte le gène $B$, barrure liée au sexe ;

- I e caractère argenté n'est pas étendu à toute la variété, nous avons pu déceler et fixer des animaux dorés ss ;

- D'une façon très variable, les Marans portent divers gènes de dessin de la plume, tels que Sg, Pg et As ;

- Les animaux étudiés n'étaient pas tous purs pour E. Nous avons trouvé des génotypes probablement $\mathrm{E}_{1} e^{+}$, ou du moins contenant un allèle de $\mathrm{E}$, récessif de $\mathrm{E}$ et de $e$ et se manifestant par un phénotype noir ;

- I e gène $\mathrm{E}$ semble lié à un facteur létal ;

- Ia Marans porte un facteur de décoloration retardée, la Sussex ayant apporté inversement un facteur de coloration retardée, probablement Wh.

En résumé, la Marans coucou argentée ne doit pas être sélectionnée d'après la conformité au standard, mais travaillée et fixée pour le génotype EE B - S - Nous pensons qu'il doit être facile de tester des coqs Marans avec des poules Rhode Island rouge avant la saison de reproduction, les coqs gardés devant avoir donné uniquement des enfants de type Marans.

3. - Enfin, les animaux analysés offrent la possibilité de créer une souche de génotype $e^{+} e^{+} \mathrm{BB}$ dorée ou argentée qui serait autosexable. Si l'on ne peut obtenir des animaux tels ayant un sous-plumage blanc, la possibilité demeure de substituer le gène $e$ au gène $e^{+}$. Dans ce cas, la souche ainsi créée ne serait pas autosexable.

C'est dans ce sens que nous poursuivons notre travail.

\section{BIBI,IOGRAPHIE}

Cochez (L. P.). - Communications personnelles, I954.

Hut' (F. B.). - Genetics of the fowl, p. I62-22I, McGraw-Hill, New-York, I 949 .

HuIT (F. B.). - Lethal action of the gene for extension of black pigment in the fowl. Genetics, 36, 3, p. 2I3-234, I95I. 
Pease (M.), Cock (A.). - Coloration retardée. Un gêne récessif affectant la couleur et le dessin du duvet. IXe Congrès Mondial d'Aviculture, 1, p. 67, Paris, I95I.

Kimbais (E.). - Genetic relation of extended black to wild type plumage pattern in the fowl. Poultry Science, 31, I, p. 73-79, I952.

Kimbal (E.). - Genetics of secondary plumage patterns in the fowl. Poultry Science, 32, I, p. I3-I7, I953.

Krmball (E.). - Genetics of birchen plumage pattern in the fowl. Poultry Science, 33, 3, p. 472-48I, I954.

Marcellin (R.). - La poule de Marans. B. T. I., 57, p. 209-222, I95I.

I.N.R.A.

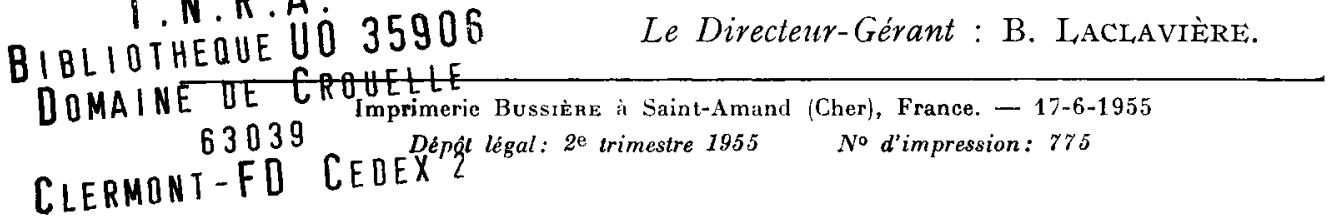

Le Directeur-Gérant : B. LACLAviÈRE. 